\title{
La modernisation du système CRISTAL de gestion des crues et des étiages du bassin de la Loire
}

\author{
The CRISTAL system of floods and low water management in the Loire \\ basin : the modernization
}

par L. Moulin

DIREN Centre, Service de Bassin Loire-Bretagne

R. Thépot

Etablissement Public d'Aménagement de la Loire et de ses Affluents

The basin of the river Loire in France covers $115000 \mathrm{~km}^{2}$. For floods prevention, water ressources management, and more generally for a permanent monitoring of the environment, a system of hydrological telemetric stations is under operation since 1985 on the river and its tributaries. Data from more than 150 stations are sent to 8 centers for processing calculation, and decision regarding reservoir management, flood warming, and operationnal hydrology monitoring.

To maintain reliability, and satisfy new needs, a renovation of the equipment and software is actually conducted. A first investment of 36.6 millions FF has started in 1996.

Those expenses cover the telemetry stations (with extension to 210 stations) the communication system (telephone, radio, satellite), and the supervision system.

Part of the operation includes advanced data treatments dedicated specifically to decision support, namely: hydrological model, meteorological radar image processing integration of information from heterogeneous sources, reporting and dissemination of information.

\section{I — LE SYSTEME DÉJÀ EN PLACE}

\subsection{Une utilisation commune à plusieurs besoins}

Mis en service en 1985, le réseau automatique de collecte de données hydrologiques et météorologiques du bassin de la Loire a permis depuis plus de dix ans d'assurer la gestion des ouvrages de Villerest et Naussac en crue et en étiage, et d'effectuer l'annonce des crues sur le bassin de la Loire à l'amont de Tours puis sur le cours principal du fleuve jusqu'à la zone sous influence maritime.

Les informations venant de 150 stations de mesure de hauteur d'eau en rivière (limnimètre) ou de pluviométrie sont transmises directement vers 8 centres d'exploitation où elles sont traitées.

Le centre principal d'Orléans La Source, établit les consignes pour les réservoirs de Villerest situé sur la Loire à l'amont de Roanne (barrage d'écrêtement de crue et de soutien des étiages) et de Naussac situé sur un affluent de l'Allier dans le département de la Lozère à proximité de Langogne (retenue de soutien des étiages uniquement).

Ce centre est également responsable du bon fonctionnement de l'ensemble du matériel et du système.
Sept autres centres, Le Puy-en-Velay, St-Etienne, Clermont-Ferrand, Moulins, Nevers, Bourges, et Orléans, établissent les messages d'annonce de crue qui sont expédiés vers les préfectures. Ces informations servent de base aux décisions préfectorales pendant les crues, et à l'établissement des messages destinés aux maires des communes riveraines en cas d'alerte.

\subsection{En service depuis plus de dix ans}

Ce système a été mis en place entre 1982 et 1985 . Il permet d'assurer la transmission automatique des mesures des hauteurs d'eau en rivière et des pluies vers plusieurs centres d'exploitation.

Le système utilise des transmissions par radio et téléphone pour les liaisons avec les stations, et Transpac (réseau filaire de transmission de données) pour les liaisons entre les centraux, avec des redondances et des configurations de secours afin de garantir la permanence de la surveillance et de l'action.

Reçues dans les centres, les informations peuvent être visualisées sous forme de courbes, de tableaux, ou sur des cartes, pour aider les opérateurs à établir un diagnostic de la situation. En cas de dépassement de seuil, ces centraux assurent l'alerte des agents d'astreinte. 
A l'aval de ces équipements de surveillance de l'état hydrologique, les opérateurs disposent aussi de modèles de prévision et d'outils d'aide à la gestion pour prendre les décisions et établir leurs consignes.

La maintenance et l'exploitation hydrologique quotidienne est assurée par 15 personnes. Le coût de fonctionnement est de l'ordre de 9 MF par an.

Ainsi, depuis 1985 , la veille a été permanente 24 h sur 24 , 365 jours par an.

Les 13 années de fonctionnement du système ont montré un niveau très satisfaisant de fiabilité permettant de valider le principe même d'une gestion de crise à l'aide d'outils élaborés fonctionnant en temps réel.

L'ensemble a connu peu d'évolution depuis sa mise en place. La durée de vie varie d'un matériel à l'autre, à la fois pour des raisons matérielles et fonctionnelles. Ainsi, les équipements les plus proches du terrain (dans notre cas, les capteurs), ont la durée de vie la plus longue. A l'autre bout de la chaîne, les centraux de traitements sur lesquels travaillent les opérateurs, doivent s'adapter plus souvent ; ils fournissent des fonctions plus élaborées d'analyse et de présentation de situations dont le taux de renouvellement ou d'adaptation est lié aux changements des besoins des utilisateurs directs ou indirects.

\subsection{La fiabilité atteinte sur le système actuel}

Pour les transmissions de données, une étude [1] a montré que le taux global de fiabilité est de l'ordre de 0,95 avec de fortes fluctuations suivant la période. Il est également apparu $[1,2]$ que les réseaux filaires pour la transmission des données n'offraient pas une garantie de fonctionnement suffisante en période de crue que ce soit en zone de montagne connaissant des crues éclairs ou plus à l'aval sous influence des perturbations océaniques : foudre, inondations des installations supportant les réseaux de transmission, arrachement des câbles...

De la même façon, les capteurs traditionnels pour la mesure des hauteurs d'eau en rivière sont assez fragiles en période de très fortes crues. Des essais sont actuellement en cours pour valider sur les hauts bassins l'utilisation de capteurs radar pour la mesure limnimétrique.

Pour assurer un niveau de fonctionnement global " tout temps ", y compris lorsque certaines infrastructures publiques ne fonctionnent plus, il est nécessaire de développer nombreuses redondances ou solutions de repli.

Après réception et validation des données, celles-ci servent de base aux prévisions. Ainsi, la gestion du réservoir de Villerest nécessite l'utilisation d'un modèle de prévision des débits à l'entrée de l'ouvrage utilisant à la fois une modélisation pluie/débit et de la propagation. Ces modèles sont alimentés en temps réel par le réseau de collecte de données. Le calcul est effectué toutes les 2 heures pendant l'événement. Les prévisions pour le bassin versant en entrée de la retenue de Villerest $\left(6500 \mathrm{~km}^{2}\right)$ ont montré un bon niveau de qualité.

\section{II — LA MODERNISATION DU SYSTÈME}

\subsection{Un renouvellement complet pour un nouveau système}

Un important programme de renouvellement du système a été lancé en 1994.
Ses objectifs prioritaires sont :

- de renouveler les équipements anciens obsolètes,

- d'augmenter la fiabilité du système tout en maîtrisant les coûts d'investissement et de fonctionnement,

- d'assurer l'intégration de la surveillance du bassin du Cher et l'extension à celui de la Maine, et l'interconnexion avec le réseau qui contrôle le bassin de la Vienne.

Au-delà de ces objectifs techniques fondamentaux, plus significatifs de l'évolution des besoins, la modernisation engagée vise également à satisfaire les demandes suivantes :

- une meilleure anticipation des événements,

- une amélioration de l'information des usagers,

- une prise en compte de la diversification des tâches des prévisionnistes hydrologues chargés du suivi des situations.

\subsection{Une opération d'envergure}

L'opération est incluse dans le Plan Loire Grandeur Nature. Une convention a été signée entre l'Etat et I'EPALA (Etablissement Public d'Aménagement de la Loire et de ses Affluents). Elle prévoit 36,6 millions de francs 1995 d'investissements partagés entre l'Etat, ministère de l'Aménagement du Territoire et de l'Environnement, pour $50 \%$, I'EPALA pour $27 \%$, et l'Agence de l'Eau Loire-Bretagne pour $23 \%$.

L'opération est sous maîtrise d'ouvrage de l'EPALA avec remise des équipements à l'Etat en fin d'exécution.

\section{III — ASSURER LA CONTINUITÉ DU SERVICE : LE SYSTÈME DE CONDUITE ET D'ORGA- NISATION DES DONNÉES (SCOD)}

\subsection{L'architecture générale du nouveau système de col- lecte de données}

Une étude préliminaire a été réalisée entre 1994 et 1996. pour réaliser les cahiers des charges des équipements prioritaires nécessaires à la continuité du service.

L'étude a défini l'architecture générale du nouveau réseau et l'infrastructure de télécommunication. L'architecture choisie privilégie la répartition des tâches et des ressources sur l'ensemble des sites sans point de passage centralisé. Ce choix garantit le fonctionnement de chaque centre quel que soit l'état des autres centres. Le centre principal d'Orléans bénéficie d'une supervision générale du réseau à des fins de maintenance sans que ce rôle soit pénalisant pour les autres centres.

Les fonctions de mesure, de collecte et de traitement ont été séparées afin d'assurer pour chacune leur évolutivité de manière distincte et de diminuer les coûts ultérieurs d'adaptation.

Trois lots ont ainsi été définis : les stations de mesure sur le terrain, les Unités de Collecte chargées de l'appel des stations et des liaisons avec les centraux, et des Unités de Traitement placées dans les centres d'exploitation pour assurer l'alerte et l'exploitation immédiate par les hydrologues.

Une partie importante des études préliminaires a porté sur le choix des vecteurs de transmission. Des solutions permettant une transmission entièrement hertzienne de bout en bout ont été recherchées pour éviter les réseaux filaires. Ainsi, certaines solutions GSM ou satellitaires ont dû être écartées car elles utilisaient en partie des liens câblés pour acheminer les informations. 
En fait, ce type de besoins sur une zone géographique aussi étendue (qui dépasse le niveau régional) est assez marginal en France. Des offres de transmission par satellite existent mais pour des débits d'information élevés. Elles reposent sur l'utilisation de satellites géostationnaires. Si elles peuvent être utilisées pour des liaisons entre centraux, elles ne sont pas adaptées, pour des questions de coût, aux liaisons avec les stations (les flux de données sont trop faibles).

L'apparition d'offres de télétransmission utilisant des constellations de satellites basses orbites d'un coût plus faible pourrait permettre de répondre mieux au problème.

\subsection{La mise en œuvre des premiers investissements}

Pour assurer la réalisation du SCOD, trois marchés industriels et un marché de prestations intellectuelles ont été passés en avril 1997 pour la conception, la fabrication, et l'installation des équipements des stations de mesure sur le terrain (société Centralp), du nouveau système de transmission, et des nouveaux centraux de traitement informatique installés dans les centres d'exploitation (société Cap Gemini pour ces deux lots), ainsi que pour une prestation d'assistance technique du maître d'ouvrage et de suivi des trois marchés industriels (société Euriware).

Ces marchés sont découpés en trois tranches :

- une première tranche de 18 mois pour la conception, la réalisation, et l'installation de prototypes,

- une deuxième tranche d'un an pour le déploiement sur les bassins actuellement couverts par le réseau Cristal (Loire, Allier, Cher),

- une troisième tranche d'un an pour le déploiement sur le bassin de la Maine.

La première tranche d'un an et demi se décompose entre 12 mois pour l'établissement des spécifications, la réalisation des prototypes (matériels et logiciels), et leur recette en usine, et 6 mois pour l'installation sur site et une période probatoire dite de marche industrielle consacrée à s'assurer du niveau de performance des installations.

L'installation des prototypes concernera les équipements du centre principal et du centre d'annonce de crues de Nevers ainsi que des stations sur ce secteur. L'ancien système continuera de fonctionner et restera le support de la gestion. Après la recette des prototypes, un déploiement sera ensuite effectué par secteur (découpage lié à des contraintes d'exploitation et de couverture radio), le basculement de la zone concernée de l'ancien système vers le nouveau deviendra alors définitif. C'est une phase critique du projet qui nécessite une bonne organisation de la logistique pour la livraison des matériels, leur installation, et leur connexion. La gestion sera effectuée sur le nouveau système dès que possible. Le déploiement est prévu pour durer un an, entre octobre 1998 et octobre 1999.

\subsection{L'acquisition des données sur le terrain}

La plupart des capteurs limnimétriques et pluviométriques restent en place. Seules les électroniques d'acquisition des données sont changées.

Ces stations d'acquisition sont conformes au cahier des charges du référentiel technique " PLQ 2000 ". Ce standard technique sert de base à une certification que le ministère de l'Aménagement du Territoire et de l'Environnement met en place actuellement pour les équipements d'hydrométrie.
Cet appel d'offres est le premier en France à y faire référence. Ce référentiel s'appuie sur une norme européenne de transmission de données (ETSI 300-230). Il concerne non seulement les stations de mesure mais aussi les systèmes informatiques qui doivent dialoguer avec.

\subsection{La transmission des données}

Les liaisons avec les stations sont assurées par des unités de collecte qui prennent en charge l'appel des stations et transmettent les informations vers les centres de traitement. Elles reçoivent aussi les alarmes venant des stations appelantes. Ces tâches sont effectuées par un PC compatible de qualité industrielle utilisant le système d'exploitation Microsoft Windows NT auxquels sont adjoints des équipements de transmission (téléphone, radio, RNIS, et satellite). Ce matériel fonctionne de manière autonome sans opérateur. Il est contrôlable à distance.

Les transmissions locales avec les stations, qui supportent des flux faibles, sont assurées par le lien radio à l'amont (la rareté des fréquences radio disponibles a empêché son extension à l'aval) ou le réseau téléphonique. Des solutions satellitaires ont été étudiées mais étaient d'un coût prohibitif. Les transmissions longues distances, aux flux de données plus importants, sont assurées par un réseau satellite Vsat doublé par Numéris.

\subsection{Le traitement des données}

L'architecture informatique des centraux est de type Client/Serveur. Ils utilisent le système d'exploitation Microsoft Windows NT pour le serveur, Microsoft Windows 95 pour les postes client, et une base de donnée Oracle.

Il est prévu que le centre principal traite 250 stations pouvant être appelées toutes les demi-heures.

Le mécanisme d'alerte pourra gérer des alarmes composites déclenchées sur croisement d'informations.

L’opérateur accède au système via quatre ensembles fonctionnels :

- la supervision pour l'exploitation hydrologique en temps réel,

- l'exploitation des données mémorisées pour le temps différé,

- le rejeu pour les simulations d'épisodes hydrologiques à des fins d'étude ou de formation,

- l'administration du système pour la maintenance et le suivi du fonctionnement.

La supervision temps réel a été isolée de façon à assurer des temps de réponse courts. La visualisation se fera sous forme de cartes (fonds de carte créés avec le logiciel ArcView), de synoptiques, et plus classiquement de tableaux et de courbes. Le système assure l'édition automatique de rapports préformatés (message de crue...) incluant des données collectées, produits à l'aide du logiciel Crystal Report.

La disponibilité exigée contractuellement en moyenne annuelle est de $99,5 \%$ dans les centres d'annonce de crue, et de $99,8 \%$ au centre principal d'Orléans La Source avec un temps maximum d'indisponibilité de $4 \mathrm{~h}$.

La mise en œuvre du nouveau système va avoir un fort impact sur la maintenance. La plupart des nouveaux équipements seront administrables à distance avec supervision, configuration, et chargement des logiciels. Par l'enregistrement systématique des incidents sur le réseau, un suivi plus fin de pannes sera également possible permettant de concen- 
trer les efforts sur les points fragiles. L'automatisation d'une partie des tâches de bilan des opérations, un partage des informations relatives au fonctionnement et un meilleur accès pour tous les agents concernés facilitera les diagnostics et les interventions.

\section{IV — VERS LA MISE EN PLACE D'UN SYSTÈME D'AIDE POUR LES PRÉVI- SIONS ET LES DÉCISIONS (SAPD)}

\section{- 4.1. Améliorer l'information des usagers et des riverains}

L'amélioration du système de collecte de données se doit d'être complétée par des actions significatives quant à leur exploitation et à leur diffusion.

Il faut assurer les tâches qui transformeront une information partielle et globale en une information exploitable directement au niveau local.

On souhaite aussi développer l'information du grand public. La dissémination d'information hydrologique en temps quasi réel à un public assez large est un sujet assez nouveau en France surtout si il s'agit d'informations élaborées comportant des prévisions.

Plusieurs études préliminaires sont en cours aussi bien pour préciser les besoins que pour définir le cadre informatique général.

\subsection{Un changement dans l'utilisation des systèmes informatiques}

Les élus des communes et leurs services techniques, les services préfectoraux, les gestionnaires des services publics ainsi que les gestionnaires de rivières sont aujourd'hui demandeurs d'informations plus directement exploitables et d'une fiabilité encore accrue.

L'accès à des informations formulées suivant un code technique précis ne suffit plus. Désormais, une base de données ou un logiciel scientifique ne doit plus se contenter d'apporter un service ponctuel et de premier niveau (calcul, dessin, stockage de données...) mais doit tendre à :

- devenir un véritable assistant pour son utilisateur direct, s'intégrant bien dans son contexte de travail et s'adaptant à ses tâches quotidiennes,

- offrir une véritable valeur ajoutée au travail, pas seulement sur un plan quantitatif (rapidité de calcul, gestion de grandes quantités d'informations...) mais aussi et surtout qualitatif, c'est-à-dire une aide adaptée aux utilisations qui seront faites des résultats, après leur diffusion.

En dernier ressort, le phénomène physique de crue ou d'étiage nous intéresse par rapport à des préoccupations de sécurité des populations et des biens, de gestion de l'eau, de l'environnement etc.

On vise donc in fine un système d'aide à la tâche quotidienne de l'ingénieur d'étude et, du gestionnaire, mais aussi, du décideur, et progressivement, d'information du grand public. L'approche des problèmes, les connaissances et les objectifs de chacun de ces acteurs sont évidemment très variables ; ils reposent néanmoins sur l'analyse des mêmes phénomènes et des mêmes données.

Notre objectif est de concevoir un système avec des interfaces de communication adaptées aux différents interlocuteurs : type bureau d'études pour le spécialiste, outil d'aide au suivi, au diagnostic et à la décision pour les Préfets et les Elus, outil de présentation simplifiée des résultats et des conclusions pour le grand public.
Un certain nombre d'éléments existent déjà, à prendre tels quels ou à adapter, d'autres sont totalement à créer.

\subsection{Une première démarche vers les utilisateurs}

Une étude est actuellement lancée pour connaître sur des sites expérimentaux la manière d'améliorer l'information en période de crue sans changer l'aspect organisationnel du processus d'annonce de crues.

On attend de cette étude la définition des outils facilitant l'utilisation des données transmises. Il peut s'agir d'un jeu de cartes d'inondation se référant à des côtes annoncées...

\section{DA STRATÉGIE DE DÉVELOPPEMENT DU SYSTÈME D'AIDE POUR LES PRÉVISIONS ET LES DÉCISIONS}

\subsection{Les éléments existants pour la constitution du SAPD}

Les préoccupations à l'origine de ce projet se retrouvent ailleurs. A titre de référence, on peut citer les produits suivants dans le domaine de l'hydrologie :

- l'outil SOPHIE de la DIREN Midi-Pyrénées,

- la plate-forme MISTERE développée par la société LHF, utilisée dans le cadre du projet " crues éclairs " de la Direction de l'Eau, et installée en Chine pour la gestion des crues à Shanghaï.

Il existe également de nombreux outils de type " tableaux de bord "qui ont été développés pour la gestion des ressources en eau.

Une base de travail est aussi opérationnelle sur la Loire. Avant la lettre, certains développements effectués au moment de la mise en place du premier système relevaient des approches décrites ci-avant.

Les développements à faire devront tenir compte de ces outils et du retour d'expérience qu'ils nous apportent.

L'utilisation de nouveaux modèles de prévision tels que celui développé actuellement à des fins d'étude pour la Loire moyenne doit être envisagée. Elle permettrait en effet d'effectuer des prévisions en se calant sur la situation réelle (hydrogramme constaté, brèches dans les endiguements...) et pas sur des scénarios calculés à l'avance et ainsi de simuler les évolutions possibles. Les capacités de calcul des ordinateurs font que ce qui ne pouvait pas être utilisé en temps réel peut maintenant l'être. Cependant, le problème se pose davantage au niveau de l'exploitation des résultats qui pourrait être difficile et exigerait un niveau d'expertise pas nécessairement disponible au moment de la crue. Un travail important de réflexion sur ce thème est en cours. On pense ainsi à une aide extérieure qui pourrait être sollicitée à distance pendant les événements.

\subsection{Une approche d'intégration}

Le système d'aide aux prévisions et aux décisions résulte donc du développement et de l'adaptation de nombreux outils :

- l'évaluation des précipitations par établissement de courbes isohyètes ou de lames d'eau à partir des données pluviographiques et radar, pour une meilleure anticipation par la dynamique des phénomènes pluvieux,

- modélisation des écoulements : modèles hydrologiques “ pluie-débit ruissellé " et modèles hydrauliques de propagation des débits, 
- modèles hydrauliques de simulation des champs d'inondations à partir des hauteurs et débits des cours d'eau, des niveaux attendus et de modèles numériques de terrain, pour fournir aux maires des bases qui pourront être affinées et adaptées localement pour mieux traduire les niveaux de préalerte et d'alerte,

- supports documentaires ou informatiques pour l'analyse des situations (d'inondation, d'étiage, ou présentant d'autres caractères exceptionnels), puis pour l'aide à la décision.

Ces outils exploiteront les informations recueillies par le réseau de collecte et d'organisation des données.

En les reliant, le système final permettra :

- de croiser plus facilement des informations de différentes sources,

- d'offrir des interfaces utilisateurs cohérentes pour le poste de travail de prévisionniste,

- de développer les outils d'aide à la décision à partir de l'exploitation des diverses sources,

- d'avoir des informations élaborées pour une meilleure diffusion et répondant mieux aux besoins.

\subsection{Le processus de mise en place}

Dans le cas du SAPD, certains concepts sont nouveaux ; en fonction de ce constat, on peut d'ores et déjà prévoir un allongement dans le temps pour sa réalisation.

Une politique de partenariat a été développée ; elle vise :

- à s'assurer l'appui d'experts dans des domaines très spécialisés,

- à favoriser l'émergence de concepts nouveaux par la collaboration de plusieurs équipes aux expériences diverses.

Deux projets sont suivis en parallèle avec ceux en cours sur le Loire :

- le projet [3] d'outil de gestion intégré de bassin versant en cours d'étude au Service Technique Central des Ports Maritimes et des Voies Navigables,

- le projet de plate-forme " Crues Eclairs " réalisée pour la Direction de l'Eau du ministère de l'Aménagement du Territoire et de I'Environnement.

\subsection{Une montée en puissance progressive pour gérer} la période transitoire

Le SAPD sera mis en place progressivement, ne serait-ce que si des outils sont opérationnels, pour effectuer la gestion des crues et des étiages qui pourraient arriver d'ici le complet déploiement (les crues de juillet 1997 en Europe de l'Est sur l'Oder ou l'étiage 1997 en France sont là pour prouver cette constante pression). Dans I'hypothèse où aucun événement ne surviendrait, ces investissements nous aideront à former les équipes à ces nouveaux concepts.

D'ores et déjà, les centres d'annonce de crues qui couvrent l'amont du bassin (Le Puy-en-Velay, Saint-Etienne, et Clermont-Ferrand) ainsi que le centre principal d'Orléans sont équipés du terminal Météotel qui leur permet de recevoir par satellite de Météo-France des images satellite, radar, et des informations du réseau sol permettant de connaître la situation météorologique. En complément de la mise en service du radar météorologique de Sembadel qui couvre les hauts bassins, ces mêmes centres ont été équipés du logiciel Calamar de la société Rhéa qui, par réception directe des images de ce radar et croisement de cette information avec les données de pluviomètres du réseau Cristal, fournit des lames d'eau sur la zone amont (bassin versant total de $20000 \mathrm{~km}^{2}$ avec un découpage en sous bassins).

Les délais de mise en place du SAPD sont les suivants : - juin 1998, fin de la phase de définition,

- décembre 1998, fin de la phase d'équipement préliminaire,

- décembre 2001, fin de la mise en place.

\section{RÉFÉRENCES}

[1] L. Moulin, D. Reinbold, Le réseau de collecte hydrométéorologique du bassin de la Loire : bilan de l'exploitation et enseignement sur la fiabilité, journées de la SHF, Nîmes, 14-16 septembre 1994.

[2] Z. Gasowski, O. Norotte, N.G. Camphuis, rapport de mission, expertise française en Pologne suite au inondations de l'Oder et de la Vistule, août 1997.

[3] G. Morel. Réutilisation et intégration de codes de calcul dans les systèmes à base de connaissances, application aux systèmes de conduite avancée de bassins versants, rapport de DEA de l'Université de Technologie de Compiègne, septembre 1997. 